\title{
Kaon-Nucleon potential from lattice QCD
}

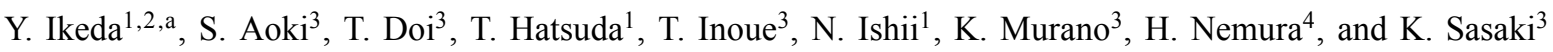

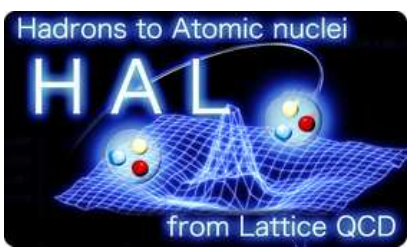

1 Depertment of Physics, The University of Tokyo, Tokyo 113-0033, Japan.

2 Nishina Center for Accelerator-Based Science, Institute for Physical and Cemical Research (RIKEN), Wako, Saitama 351-0198, Japan

3 Depertment of Pure and Applied Sciences, The University of Tsukuba, Tsukuba, Ibaraki 305-8577, Japan

4 Depertment of Physics, Tohoku University, Sendai, Miyagi 980-8578, Japan

\begin{abstract}
We study the $K N$ interactions in the $I\left(J^{\pi}\right)=0\left(1 / 2^{-}\right)$and $1\left(1 / 2^{-}\right)$channels and associated exotic state $\Theta^{+}$from $2+1 \mathrm{f}$ avor full lattice QCD simulation for relatively heavy quark mass corresponding to $m_{\pi}=$ $871 \mathrm{MeV}$. The s-wave $K N$ potentials are obtained from the Bethe-Salpeter wave function by using the method recently developed by HAL QCD (Hadrons to Atomic nuclei from Lattice QCD) Collaboration. Potentials in both channels reveal short range repulsions: Strength of the repulsion is stronger in the $I=1$ potential, which is consistent with the prediction of the Tomozawa-Weinberg term. The $I=0$ potential is found to have attractive well at mid range. From these potentials, the $K N$ scattering phase shifts are calculated and compared with the experimental data.
\end{abstract}

\section{Introduction}

First evidence of the $\Theta^{+}(1540)$ (an exotic state with baryon number $B=1$ and strangeness $S=+1$ which corresponds to the quark content $u u d d \bar{s}$ ) has been reported by the LEPS Collaboration at SPring-8 [1]. Although numbers of experimental studies have been performed since then, the existence of the $\Theta^{+}(1540)$ is still controversial: CLAS Collaboration observed no signal from their high statistic data [2], and other experiments at high energies with high statistics and good mass resolutions did not $\mathrm{f}$ nd positive evidence as reviewed in [3]. On the other hand, LEPS Collaboration and DIANA Collaboration have recently reconf rmed the $\Theta^{+}(1540)$ signal with high statistics $[4,5]$. They indicate that the production mechanism of the $\Theta^{+}(1540)$ might be highly reaction dependent if it exits in nature.

Theoretically, QCD studies of the mass and the quantum numbers of the $\Theta^{+}(1540)$ have been attempted using lattice QCD simulations and QCD sum rules. In the lattice QCD studies with the quenched approximation, existence of the low-mass pentaquark is not conclusive yet (see e.g. the summary given in [6]). Recent QCD sum rule studies [7] with the operator product expansion up to dimension 14 show some candidates of the $\Theta^{+}(1540)$ in the $I\left(J^{\pi}\right)=0\left(1 / 2^{-}\right), 1\left(1 / 2^{-}\right), 0\left(3 / 2^{+}\right)$and $1\left(3 / 2^{+}\right)$channels.

\footnotetext{
${ }^{a}$ e-mail: yikeda@nt.phys.s.u-tokyo.ac.jp
}

Determination of the quantum numbers of $\Theta^{+}(1540)$ was also attempted from the phase shift analyses of the $K N$ scattering data, and some candidates in $L_{2 I, 2 J}=D_{03}, F_{05}$ were reported [8].

The main purpose of the present study is to investigate the low energy $K N$ potentials in the $I\left(J^{\pi}\right)=0\left(1 / 2^{-}\right)$ and $1\left(1 / 2^{-}\right)$channels and to shed new light on $\Theta^{+}$from the $2+1$ f avor full QCD simulations. A systematic method to extract the hadron-hadron potential from the equal-time Bethe-Salpter amplitude measured on the lattice has been recently developed and applied to the nucleon-nucleon potential by HAL QCD Collaboration [9-13] The potential obtained in this method can be utilized to calculate the scattering observables and to study the resonances and bound states. We utilize this method to extract the $K N$ potentials and phase shifts.

This paper is organized as follows. In section 2 , the formalism to extract the $K N$ potential from lattice QCD is brief y reviewed. Our numerical setup of the lattice QCD simulation is then shown in section 3 , and the results are shown in section 4 . These results are discussed in section 5 , and summary is given in section 6 . 


\section{Formalism}

Following the basic formulation to extract the nucleonnucleon interaction $[9,10]$, we brief y show the equations to obtain the $K N$ potentials below. We start with an effective Schrödinger equation for the equal-time Bethe-Salpeter (BS) wave function $\phi(\mathbf{r})$ :

$$
-\frac{\nabla^{2}}{2 \mu} \phi(\mathbf{r})+\int d \mathbf{r} U\left(\mathbf{r}, \mathbf{r}^{\prime}\right) \phi\left(\mathbf{r}^{\prime}\right)=E \phi(\mathbf{r})
$$

where $\mu\left(=m_{K} m_{N} /\left(m_{K}+m_{N}\right)\right)$ and $E$ denote the reduced mass of the $K N$ system and the non-relativistic energy, respectively. The non-local potential $U\left(\mathbf{r}, \mathbf{r}^{\prime}\right)$ can be expanded in powers of the relative velocity $\mathbf{v}=\nabla / \mu$ at low energies,

$$
\begin{aligned}
U\left(\mathbf{r}, \mathbf{r}^{\prime}\right) & =V(\mathbf{r}, \mathbf{v}) \delta\left(\mathbf{r}-\mathbf{r}^{\prime}\right) \\
& =\left(V_{L O}(\mathbf{r})+V_{N L O}(\mathbf{r})+\cdots\right) \delta\left(\mathbf{r}-\mathbf{r}^{\prime}\right),
\end{aligned}
$$

where the $N^{n} L O$ term is of order $O\left(\mathbf{v}^{n}\right)$. In the leading order, we have

$$
V(\mathbf{r}) \simeq V_{L O}(\mathbf{r})=\frac{\nabla^{2}}{2 \mu} \phi(\mathbf{r})+E .
$$

In order to obtain the BS wave function of the $K N$ system on the lattice, let us consider the four-point correlator:

$$
\begin{gathered}
\mathcal{G}_{\alpha}\left(\mathbf{x}, \mathbf{y}, t-t_{0} ; J^{\pi}\right)=\left\langle 0\left|K(\mathbf{x}, t) N_{\alpha}(\mathbf{y}, t) \overline{\mathcal{J}}_{K N}\left(t_{0} ; J^{\pi}\right)\right| 0\right\rangle \\
=\sum_{n} A_{n}\left\langle 0\left|K(\mathbf{x}, t) N_{\alpha}(\mathbf{y}, t)\right| n\right\rangle e^{-E_{n}\left(t-t_{0}\right)},
\end{gathered}
$$

with the matrix elements

$$
A_{n}=\left\langle n\left|\overline{\mathcal{J}}_{K N}\left(t_{0} ; J^{\pi}\right)\right| 0\right\rangle .
$$

Here $\overline{\mathcal{J}}_{K N}\left(t_{0} ; J^{\pi}\right)$ denotes a source term which creates the $K N$ system with spin-parity $J^{\pi}$ on the lattice. The fourpoint correlator in Eq. (4) is dominated by the lowest energy state with total energy $E_{0}$ at large time separation $\left(t \gg t_{0}\right)$ :

$$
\begin{aligned}
\mathcal{G}_{\alpha}\left(\mathbf{r}, t-t_{0} ; J^{\pi}\right) & =\sum_{\mathbf{y}} \mathcal{G}_{\alpha}\left(\mathbf{x}, \mathbf{y}, t-t_{0} ; J^{\pi}\right) \\
& \rightarrow A_{0} \phi_{\alpha}\left(\mathbf{r} ; J^{\pi}\right) e^{-E_{0}\left(t-t_{0}\right)},
\end{aligned}
$$

with $\mathbf{r}=\mathbf{x}-\mathbf{y}$. Thus, the $K N$ BS wave function is def ned by the spatial correlation of the four-point correlator. In Eq. (6), we assume the Dirichlet boundary condition in temporal direction, so that the temporal correlation has an expnential form, $e^{-E_{0}\left(t-t_{0}\right)}$.

The BS wave function in s-wave state is obtained under the projection onto zero angular momentum $\left(P^{(l=0)}\right)$,

$$
\phi\left(\mathbf{r} ; 1 / 2^{-}\right)=\frac{1}{24} \sum_{g \in O} P_{\alpha}^{(l=0)} \phi_{\alpha}\left(g^{-1} \mathbf{r} ; 1 / 2^{-}\right),
$$

where $g \in O$ represent 24 elements of the cubic rotational group, and the summation is taken for all these elements. Using Eq. (3) and Eq. (7), we will f nd the $K N$ potential and wave function from lattice QCD.

\section{Numerical setup}

In order to calculate the $K N$ potentials in isospin $I=0$ and $I=1$ channels in $2+1$ f avor full QCD, we have utilized gauge conf gurations of JLDG(Japan Lattice Data Grid)/ ILDG(International Lattice Data Grid) generated by CPPACS and JLQCD Collaborations on a $16^{3} \times 32$ lattice [14, 15]. The renormalization group improved Iwasaki gauge action and non-perturbatively $O(a)$ improved Wilson quark action are used at $\beta=1.83$ which corresponds to the lattice spacing $a=0.1209 \mathrm{fm}$ with the $\rho$ meson mass in the chiral limit. The physical size of the lattice is about $(2.0 \mathrm{fm})^{3}$ and the the hopping parameters are taken to be $\kappa_{u}=\kappa_{d}=$ 0.1378 and $\kappa_{s}=0.1371$.

In the present simulation, we adopt the spatial wall source located at $t_{0}$ with the Dirichlet boundary condition at time slice $t=t_{0}+16$ in the temporal direction and the periodic boundary condition in each spatial direction. The Coulomb gauge fxing is employed at $t=t_{0}$. The number of gauge conf gurations used in the simulation is 700 .

\section{Results}

The masses of hadrons are obtained by ftting corresponding two-point correlators. The obtained masses of the pion, kaon and nucleon are $m_{\pi}=870.7(1.9) \mathrm{MeV}, m_{K}=$ 911.5(1.9) $\mathrm{MeV}$ and $m_{N}=1795.5(6.9) \mathrm{MeV}$, respectively. Thus the $K N$ threshold energy is $2707 \mathrm{MeV}$.

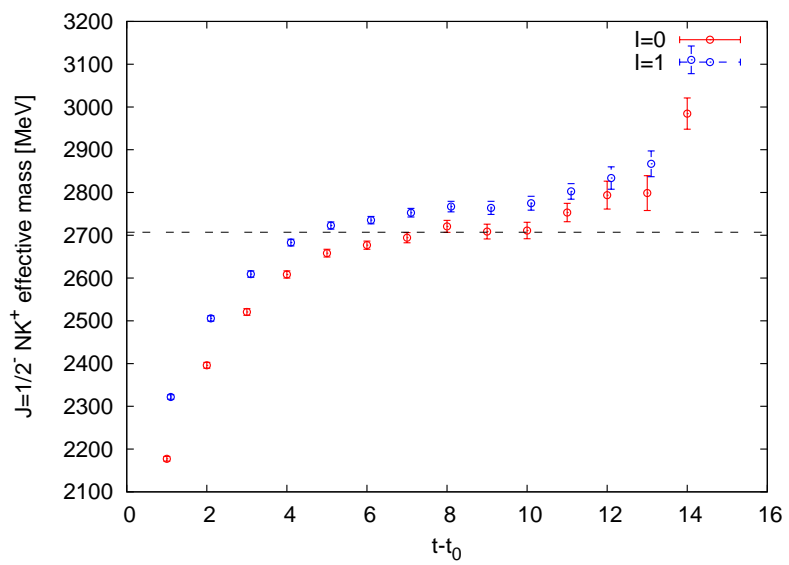

Fig. 1. Effective mass plot of the $K N$ states in $I=0($ red) and $I=$ 1(blue) channels. The dashed line denotes the threshold energy of the $K N$ measured in this simulation.

Fig. 1 shows the effective masses of the $K N$ states in the $I=0$ and $I=1$ channels together with the $K N$ threshold energy. Effective masses are obtained from the temporal correlation in Eq. (6). We observe plateaus at large time separation, $t-t_{0} \geq 7$. The best $\mathrm{ft}$ in the plateau gives $M_{I=0}=2708(11) \mathrm{MeV}$ and $M_{I=1}=2761(10) \mathrm{MeV}$, so that the lowest $K N$ state is the $I=0$ channel.

Figs. 2 and 3 show BS wave functions of the $K N$ scatterings in the $I=0$ and $I=1$ channels, respectively. They are obtained from the lattice QCD simulations at large time 


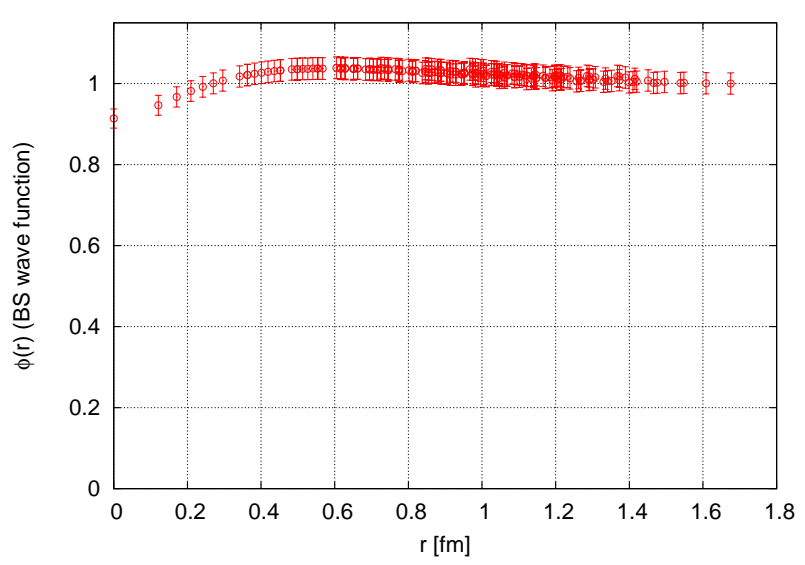

Fig. 2. The BS wave function of the $K N$ scattering in the $I=0$ channel.

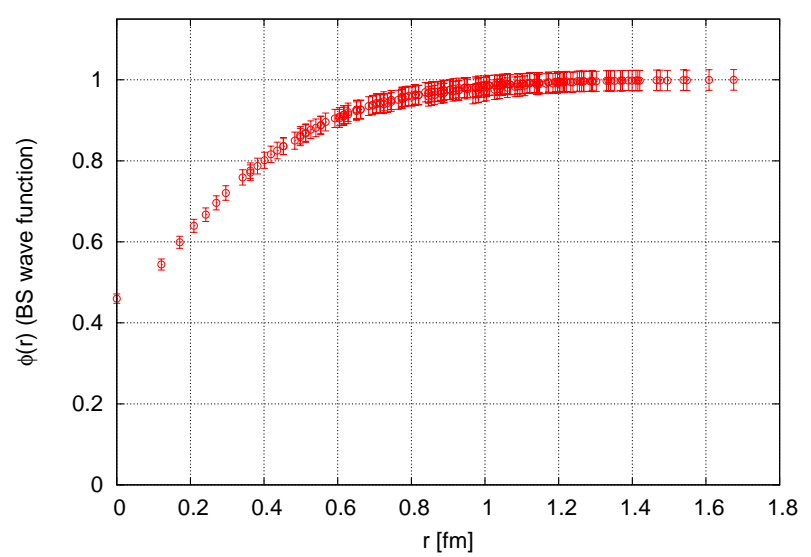

Fig. 3. The BS wave function of the $K N$ scattering in the $I=1$ channel.

separation, $t-t_{0}=8$. The large $r$ behavior of the BS wave functions in both channels do not show a sign of bound state, though more detailed analysis is needed with large volumes for a def nite conclusion. The small $r$ behavior of the BS wave functions suggests some repulsive interaction at short distance $(r<0.3 \mathrm{fm})$. Also, the repulsion in the $I=1$ channel seems to be stronger than that in the $I=0$ channel.

The potential $V(r)$ without the constant energy shift $E$ in Eq. (3) for the $I=0(I=1) K N$ state is shown in Fig. 4 (Fig. 5). These potentials are also calculated by using the data at $t-t_{0}=8$. As expected from the BS wave functions in Figs. 2 and 3, we observe the repulsive interactions at short distance in both channels. Also, we observe the attractive well in the mid range $(0.4<r<0.8 \mathrm{fm})$ in the $I=0$ channel. In the constituent quark model of hadrons [16], similar short range repulsion in $K N$ system has been predicted, while the attraction has not been found.

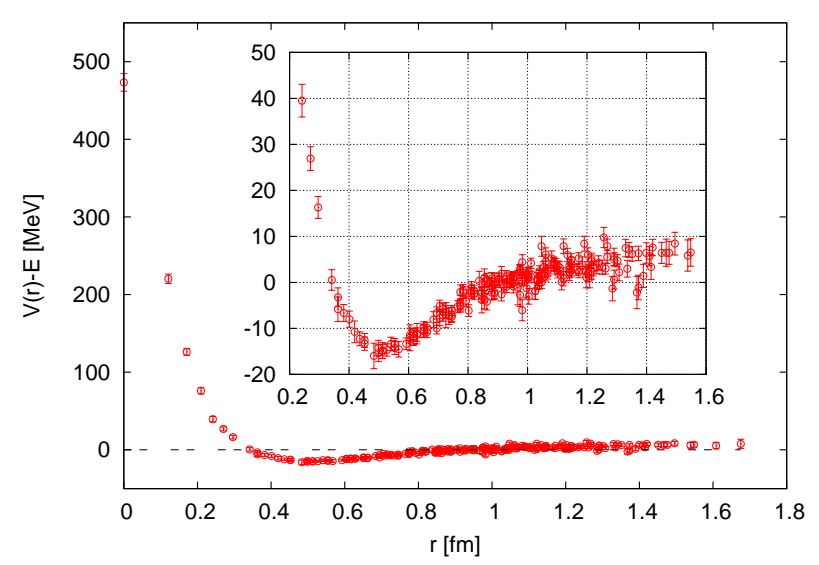

Fig. 4. The potential of the $K N$ state in the $I=0$ channel without the energy shift $E$ in Eq. (3).

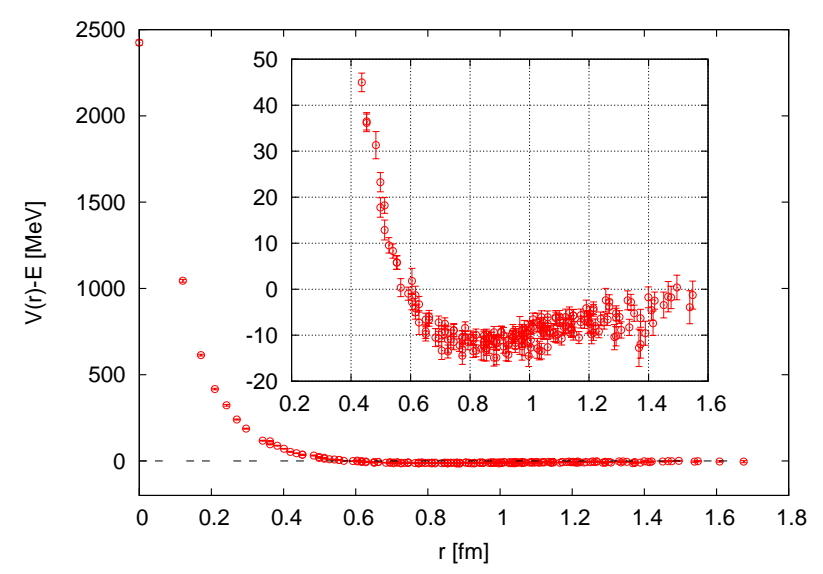

Fig. 5. The potential of the $K N$ state in the $I=1$ channel without the energy shift $E$ in Eq. (3).

\section{Discussion}

Results shown in the previous section indicate that there are no bound states in the $I\left(J^{\pi}\right)=0\left(1 / 2^{-}\right)$and $1\left(1 / 2^{-}\right)$ states for the pion mass $m_{\pi} \sim 870 \mathrm{MeV}$.

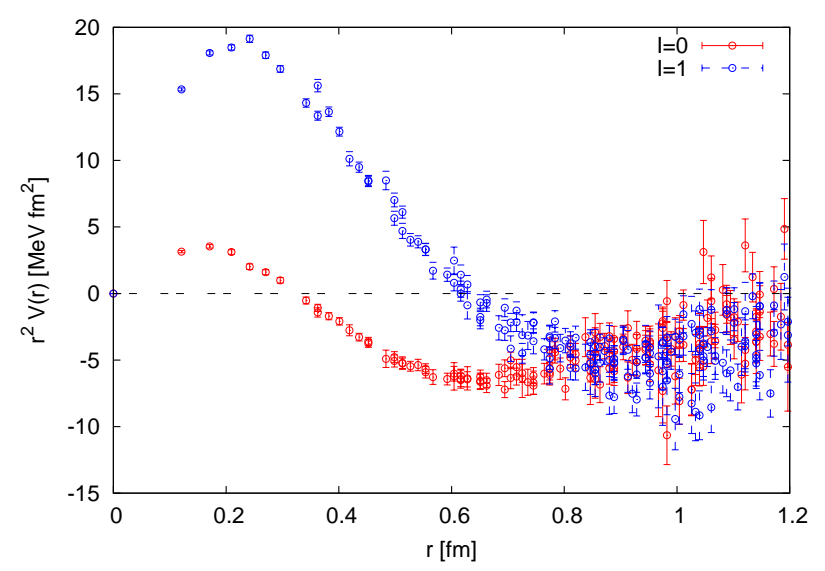

Fig. 6. The potentials multiplied by a volume factor $\left(r^{2} V(r)\right)$. 
In order to compare the strength of the repulsion between the different isospin states, we plot the potentials multiplied by a volume factor $\left(r^{2} V(r)\right)$ in Fig. 6 with the energy shifts $E=-5.0 \mathrm{MeV}$ for the $I=0$ channel and $E=5.0 \mathrm{MeV}$ for the $I=1 \mathrm{channel}$. These energy shifts are estimated from the asymptotic behavior of the potentials at large $r$, though analyses with large volumes are needed to determine these energy shifts more precisely. As seen in Fig. 6, the repulsion at short distance in the $I=1$ channel is much stronger than that in the $I=0$ channel. This is anticipated from the Tomozawa-Weinberg (TW) term in the effective chiral Lagrangian of mesons and nucleon: The $I=0$ interaction vanishes, while the $I=1$ interaction is repulsive from the contact TW interaction, which should be compared approximately with the integral of our $r^{2} V(r)$ at short distance.

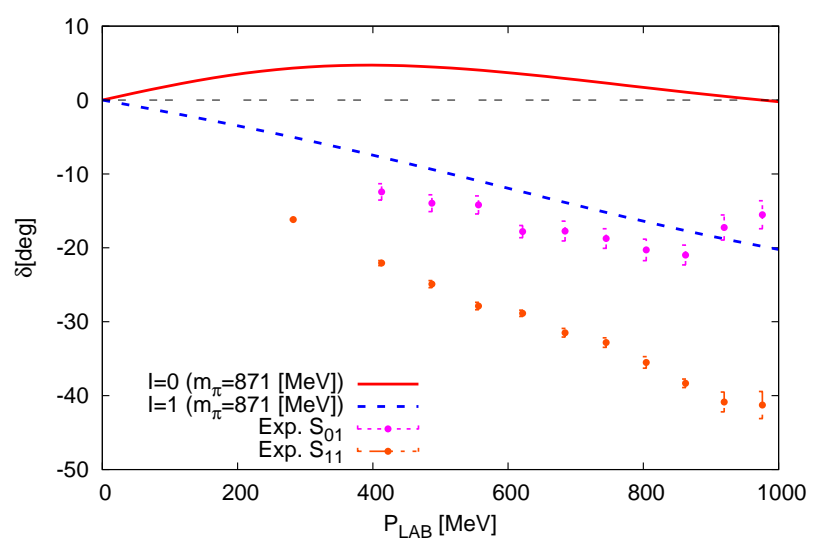

Fig. 7. The phase shifts of the $K N$ scatterings as the function of the momentum $\left(P_{\mathrm{LAB}} \mathrm{MeV}\right)$ in the laboratory frame. The red solid (blue dashed) curve shows the phase shift of the $I=0(I=1) K N$ scattering. Data are taken from Ref. [17].

By using the potentials which $\mathrm{ft}$ the lattice data in Figs. 4 and 5, we can calculate observables such as the scattering phase shifts. Fig. 7 shows such phase shifts of the $K N$ scattering together with the experimental data as a function of the laboratory momentum of the kaon. Theoretical phase shifts are calculated from the Schrödinger equation with the potentials, $V(r)$, where the energy shift $E=-5.0(5.0) \mathrm{MeV}$ in the $I=0(I=1)$ channel is taken into account. Although the hadron masses are heavy in the present simulation, qualitative behabior of the phase shifts and also the relative magnitude between the $I=0$ and $I=1$ channels are consistent with the experimental data. Simulations along this line with ligher quark masses would eventually lead to a def nite conclusion on the existence of $\Theta^{+}$in $I\left(J^{\pi}\right)=0\left(1 / 2^{-}\right)$and $1\left(1 / 2^{-}\right)$channels.

\section{Summary}

We have performed the $2+1$ f avor full lattice QCD simulation to investigate the $K N$ interaction and a possible signature of the exotic resonance $\Theta^{+}$. The s-wave $I=0$ and
$I=1$ potentials are extracted from the BS wave functions for relatively heavy quark mass corresponding to $m_{\pi}=871$ $\mathrm{MeV}$. Potentials in both channels reveal short range repulsions: Strength of the repulsion is stronger in the $I=1$ potential, which is consistent with the prediction of the Tomozawa-Weinberg term. The $I=0$ potential is found to have attractive well at mid range. From these potentials, the $K N$ scattering phase shifts are calculated and compared with the experimental data. Although the quark mass is heavy in the present simulation, the results indicate that our method is promising for future quantitative studies of the $K N$ interactions and the exotic resonances in lighter quark mass region.

We thank Columbia Physics System [18] for their lattice QCD simulation code, of which modif ed version is used in this work. The author (Y.I.) thanks Prof. M. Oka for useful discussion. This work is supported by the Large Scale Simulation Program No.0923(FY2009) of High Energy Accelerator Research Organization (KEK), the Grant-in-Aid of MEXT (No.20340047) and the Grantin-Aid for Scientif c Research on Innovative Areas (No. 2004: 20105001,20105003).

\section{References}

1. T. Nakano et al. [LEPS Collaboration], Phys. Rev. Lett. 91, (2003) 012002 [arXiv:hep-ex/0301020].

2. B. McKinnon et al. [CLAS Collaboration], Phys. Rev. Lett. 96, (2006) 212001 [arXiv:hep-ex/0603028].

3. M. Danilov and R. Mizuk, arXiv:0704.3531 [hep-ex].

4. T. Nakano et al. [LEPS Collaboration], Phys. Rev. C 79, (2009) 025210 [arXiv:0812.1035 [nucl-ex]].

5. V. V. Barmin et al. [DIANA Collaboration], arXiv:0909.4183 [hep-ex].

6. T. Doi, Prog. Theor. Phys. Suppl. 168, 45 (2007) [arXiv:0704.0959 [hep-ph]].

7. P. Gubler, D. Jido, T. Kojo, T. Nishikawa and M. Oka, Phys. Rev. D 79, (2009) 114011 [arXiv:0902.2049 [hep-ph]].

P. Gubler, D. Jido, T. Kojo, T. Nishikawa and M. Oka, arXiv:0911.2547 [hep-ph].

8. N. G. Kelkar, M. Nowakowski and K. P. Khemchandani, J. Phys. G 29, (2003) 1001 [arXiv:hep$\mathrm{ph} / 0307134]$.

W. R. Gibbs and R. Arceo, Phys. Rev. C 75, (2007) 035204 [arXiv:nucl-th/0611095].

9. N. Ishii, S. Aoki and T. Hatsuda, Phys. Rev. Lett. 99, (2007) 022001 [arXiv:nucl-th/0611096].

10. S. Aoki, T. Hatsuda and N. Ishii, arXiv:0909.5585 [hep-lat].

11. H. Nemura, N. Ishii, S. Aoki and T. Hatsuda, Phys. Lett. B 673, (2009) 136 [arXiv:0806.1094 [nucl-th]].

12. H. Nemura, N. Ishii, S. Aoki and T. Hatsuda [PACSCS Collaboration], arXiv:0902.1251 [hep-lat].

13. T. Inoue [HAL QCD Collaboration], arXiv:0911.2305 [hep-lat].

14. T. Ishikawa et al., PoS LAT2006, (2006) 181 [arXiv:hep-lat/0610050]. 
$19^{\text {th }}$ International IUPAP Conference on Few-Body Problems in Physics

15. T. Ishikawa et al. [JLQCD Collaboration], Phys. Rev.

D 78, (2008) 011502 [arXiv:0704.1937 [hep-lat]].

16. T. Barnes and E. S. Swanson, Phys. Rev. C 49, (1994) 1166.

17. K. Hashimoto, Phys. Rev. C 29 (1984) 1377.

18. Columbia Physics System (CPS), http://qcdoc.phys.columbia.edu/cps.html 